\title{
ON THE MECHANISM OF NITRATE-INDUCED ALKALOSIS. THE POSSIBLE ROLE OF SELECTIVE CHLORIDE DEPLETION IN ACID-BASE REGULATION *
}

\author{
By PAUL F. GULYASSY, $\dagger$ CHARLES van YPERSELE de STRIHOU, $\ddagger$ AND \\ WILLIAM B. SCHWARTZ
}

\author{
(From the Department of Medicine, Tufts University School of Medicine, and the Renal \\ Laboratory, Pratt Clinic-New England Center Hospital, Boston, Mass.)
}

(Submitted for publication March 13, 1962; accepted June 7, 1962)

It has recently been demonstrated that the rate of both hydrogen and potassium excretion can be acutely increased when a disproportion is created between the quantity of sodium and the quantity of penetrating anion available for reabsorption (1). This experimental condition was achieved by the infusion of neutral sodium phosphate into animals which had previously been maintained on a diet poor in sodium chloride. Such short-term observations raised the possibility that sustained changes in availability of penetrating anion might of themselves induce chronic alterations in body fluid composition. This hypothesis was supported by the subsequent observation that restriction of dietary chloride prevents correction of plasma bicarbonate elevations induced by chronic hypercapnia or administration of desoxycorticosterone acetate (DOCA) $(2,3)$. In each of the latter circumstances, however, the elevated plasma bicarbonate presumably developed as a result of direct acceleration of sodium-cation exchange and these observations, therefore, throw no light on the possible consequences of a primary loss of chloride in a normal animal. Such a loss might be expected to produce either secondary sodium (and volume) depletion, diversion of sodium reabsorption to the cation-exchange mechanism, or some combination of the two. In order to investigate this problem, chloride deficiency has been produced in dogs by the repeated infusion of nitrate, an anion known to induce chloruresis (4).

\footnotetext{
* Supported in part by grants from the National Heart Institute, National Institutes of Health, Public Health Service (H-759 and HTS-5309), and the Life Insurance Medical Research Fund.

$\dagger$ Research Fellow, The Medical Foundation, Inc. $\ddagger$ Fellow of the Belgian American Education Foundation, supported by funds from the Fonds National de la Recherche Scientifique Belge.
}

\section{METHODS}

Balance studies have been carried out on 8 healthy female mongrel dogs weighing between 12 and $18 \mathrm{~kg}$. Each day the animals were fed $30 \mathrm{~g}$ per $\mathrm{kg}$ of body weight of a synthetic diet, of previously described composition (3). The intrinsic electrolyte content of the diet was 1 $\mathrm{mEq}$ of sodium, less than $1 \mathrm{mEq}$ of chloride, and $0.1 \mathrm{mEq}$ of potassium per $100 \mathrm{~g}$. The daily intake was supplemented with approximately $5.5 \mathrm{mEq}$ per $\mathrm{kg}$ of body weight of potassium as neutral phosphate $\left(4 \mathrm{HPO}_{4}=/\right.$ $1 \mathrm{H}_{2} \mathrm{PO}_{4}^{-}$) except in period III when the electrolyte content was modified as described below. All the animals were force-fed twice daily.

Each study was initiated by a control period during which the synthetic diet was administered for a period of 6 to 10 days. The study was then divided into four periods. ${ }^{1}$

Period I. Sodium nitrate infusions. On 5 to 7 successive days the animals were infused before the morning feeding with 11 mmoles per $\mathrm{kg}$ of body weight of sodium nitrate. The sodium nitrate solution (335 mmoles per L) was administered with a constant infusion pump over a period of 90 minutes. On a few occasions the animals vomited small amounts of material (10 to $30 \mathrm{cc}$ ). In each instance the vomitus was completely recovered and re-fed later in the day.

Period II. Observations were continued on 6 dogs for a period of 5 to 6 days after nitrate infusions were discontinued. One of these animals, dog I, received a daily supplement of $40 \mathrm{mEq}$ of $\mathrm{KHCO}_{3}$ for 4 days, after which it was returned to the previous intake for 4 days.

Period III. Administration of chloride supplement. Each of 6 dogs received chloride according to one of three protocols. a) $\mathrm{NaCl}$ supplement (potassium-free intake): Three dogs, F, G, and $\mathrm{O}$, were given approximately $5.5 \mathrm{mEq}$ per $\mathrm{kg}$ of body weight of sodium as neutral phosphate in place of an equal quantity of potassium phosphate. They received, in addition, 5.5 mmoles of sodium chloride per $\mathrm{kg}$ of body weight for a period of 6 days. b) $\mathrm{KCl}$ supplement (sodium-free intake): Two dogs, $\mathrm{L}$ and $\mathrm{I}$, were given 3.5 mmoles per $\mathrm{kg}$ of body weight of potassium chloride, in addition to their

1 One dog, E, was studied only after nitrate infusions were discontinued. 
usual potassium phosphate intake, for a period of 4 days. c) $\mathrm{NaCl}$ and $\mathrm{KCl}$ supplement: One dog, E, was given 7.7 mmoles of sodium chloride per $\mathbf{k g}$ of body weight, in addition to its usual potassium phosphate intake, for a period of 4 days.

Period IV. Two dogs whose chloride deficit had been repaired on a potassium-free intake were subsequently returned to their previous electrolyte supplement of potassium phosphate. Observations were continued for a further 5 to 6 days.

The analytic methods and calculations have been described previously (3) except for the following determinations. Nitrate was determined in plasma for 5 animals and urine for 7 according to the method of Engelbrecht and McCoy (5) modified for the Technicon autoanalyzer; inorganic sulfate was determined gravimetrically as barium sulfate (6). Plasma unmeasured anion concentration, $\left(\mathrm{Na}^{+}\right)-\left(\mathrm{Cl}^{-}+\mathrm{HCO}_{3}^{-}+\mathrm{NO}_{3}^{-}\right)$, was calculated for the 5 animals in which plasma nitrate levels were determined. During the control period nitrate was undetectable in plasma. Control urine nitrate determinations, however, averaged $4 \mathrm{mEq}$ per day, representing either baseline nitrate output or presence of unknown substances reacting as nitrate with the method used. Net acid excretion is calculated as $\left(\mathrm{NH}_{4}^{+}+\right.$titratable acid) $\left(\mathrm{HCO}_{3}^{-}\right)$. Delta net acid is expressed for periods $\mathrm{I}$ and II as the change from the average output for the control period. Delta net acid for periods III and IV is expressed as the change from the average output during the last 2 to 3 days of period II.

\section{RESULTS}

At the end of the control period, average plasma concentrations were: sodium, $144 \mathrm{mEq}$ per L; chloride, $108 \mathrm{mEq}$ per L; bicarbonate, $21.5 \mathrm{mEq}$ per $\mathrm{L}$; and potassium, $4.4 \mathrm{mEq}$ per L. Plasma

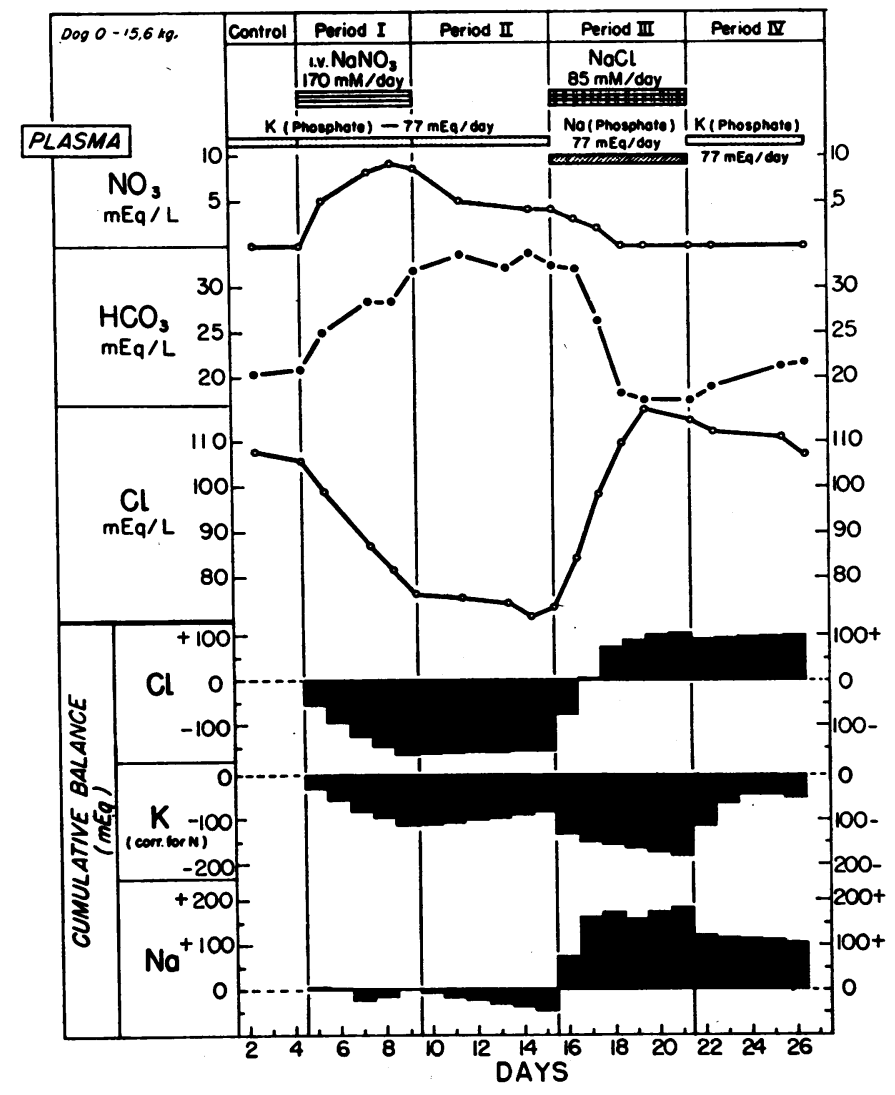

Fig. 1. Plasma composition and cumulative electrolyte BALANCES DURING A STUDY IN WHICH ALKaLOSIS WAS CORRECTED WITH SODIUM CHLORIDE. Note that correction of the alkalosis was effected while the animal was on a potassium-free diet and while potassium balance was becoming increasingly negative. The values for potassium balance have been corrected for nitrogen balance (2.7 $\mathrm{mEq}$ per $\mathrm{g}$ ). 


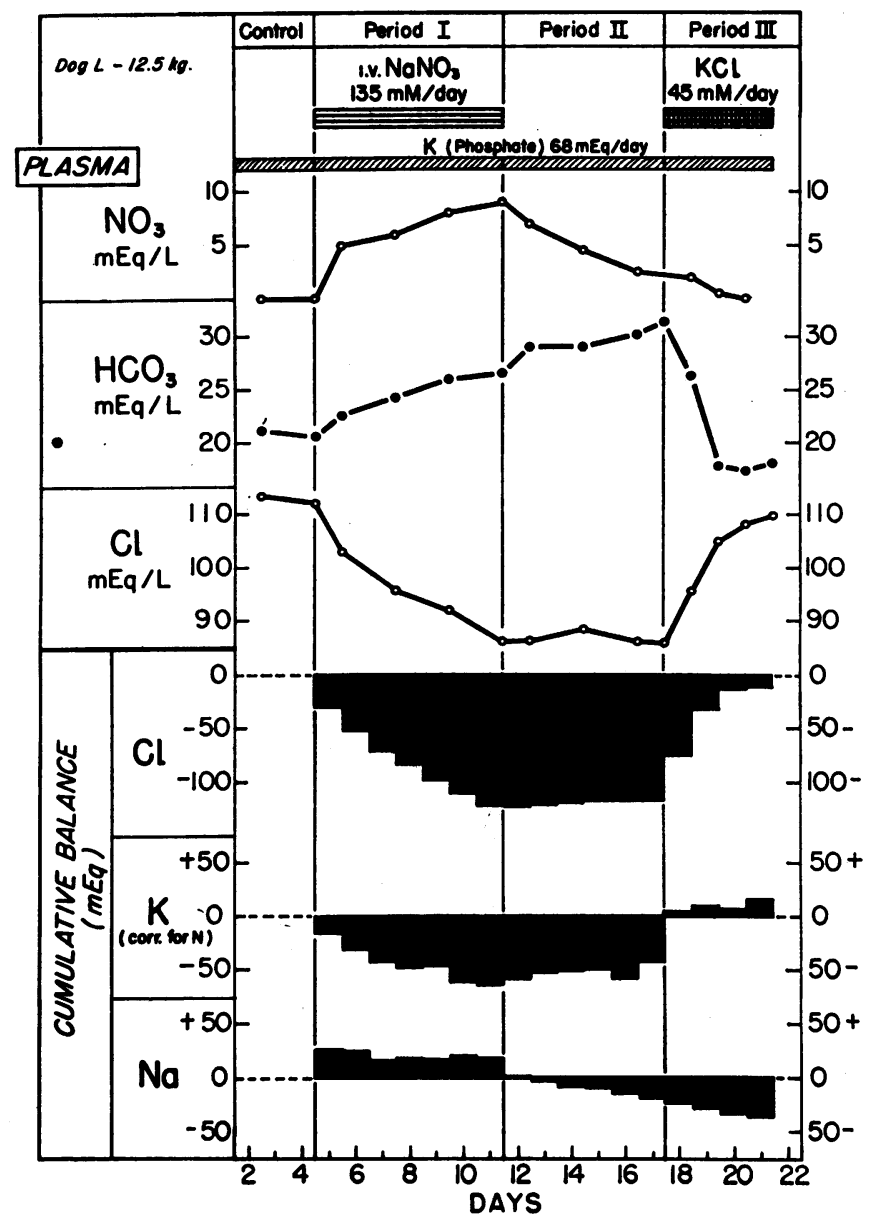

Fig. 2. Plasma composition and cumulative electrolyte BALANCES DURING A STUDY IN WHICH ALKALOSIS WAS CORRECTED WITH POTASSIUM CHLORIDE. Note that correction of the alkalosis was effected while the animal was on a sodium-free diet. The values for potassium balance have been corrected for nitrogen balance $(2.7 \mathrm{mEq}$ per $\mathrm{g})$.

$\mathrm{pH}$ averaged 7.38. Figures 1 and 2 and Tables I and II present detailed results of two representative studies. The remaining figures and table show collective data.

\section{Period I. Sodium nitrate infusions}

1. Acid-base balance. As shown in Figures 1 through 3 , plasma bicarbonate concentration rose steadily. The mean final value was $30.5 \mathrm{mEq}$ per L. As a consequence of a simultaneous rise in blood $\mathrm{pCO}_{2}, \mathrm{pH}$ rose only slightly for a final average of 7.46. The cumulative delta net acid excretion ranged from -64 to $+69 \mathrm{mEq}$. The average for the group was $-8 \mathrm{mEq}$.
2. Chloride, nitrate, unmeasured anions, and organic acids. As shown in Figures 1, 2, and 4, plasma chloride concentration fell progressively. The average final value was $79 \mathrm{mEq}$ per $\mathrm{L}$, with an average decrement of $29 \mathrm{mEq}$ per L. Chloruresis was largest on the first day and thereafter decreased steadily (Figure 5). Cumulative chloride loss ranged from 115 to $194 \mathrm{mEq}$ (Table III). Most of the $29 \mathrm{mEq}$ per $\mathrm{L}$ decrement in plasma chloride was accompanied by an increase in concentration of other anions (plasma sodium fell by an average of only $6 \mathrm{mEq}$ per L). Of this total anion increment, nitrate constituted approximately two-fifths, bicarbonate two-fifths, and unmeasured 


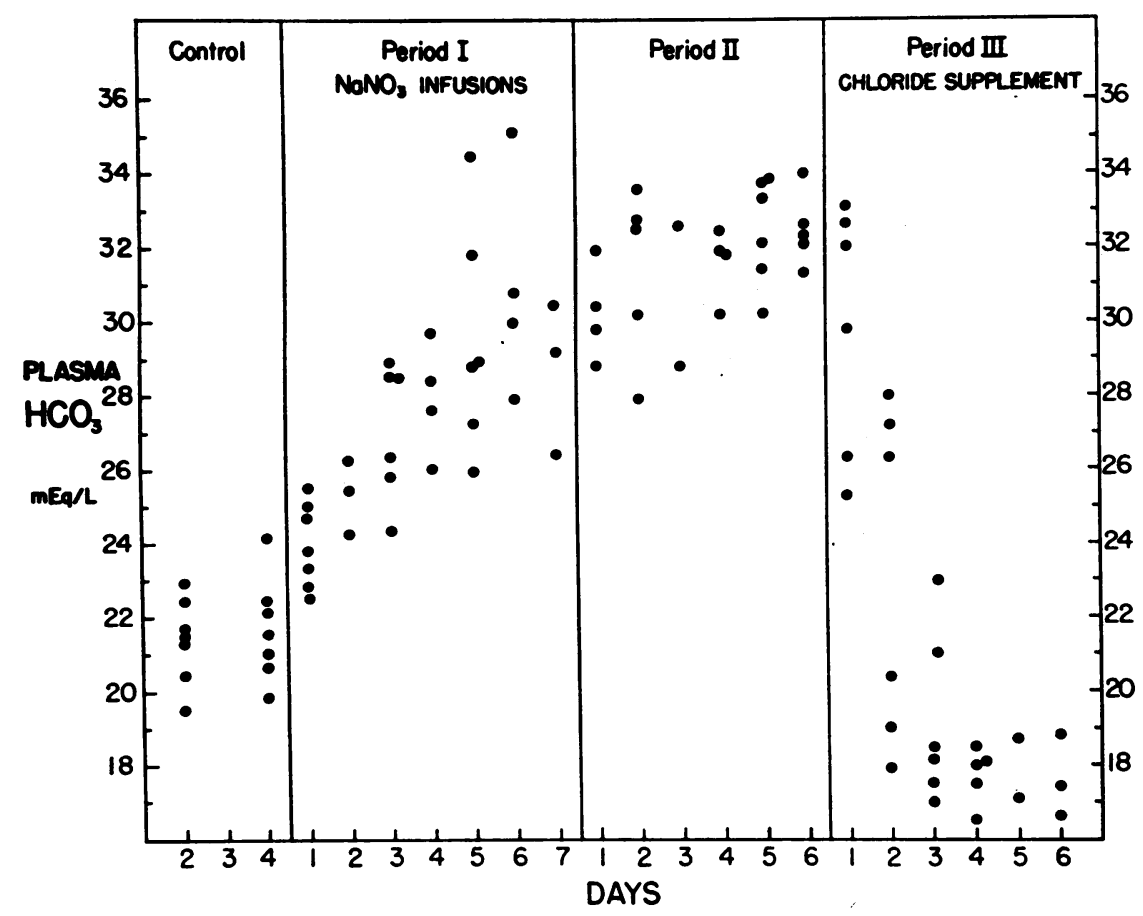

Fig. 3. Plasma bicarbonate concentrations dURing Development and repair oF NITRATE-INDUCED ALKalosis. For details of the protocols employed for provision of the chloride supplements in period III, see text. The period of $\mathrm{KHCO}_{3}$ administration in $\operatorname{dog} I$ is omitted.

anions one-fifth (Figure 6). Plasma nitrate concentration averaged $4 \mathrm{mEq}$ per $\mathrm{L}$ the morning after the first infusion and thereafter continued to rise to final levels of 6 to $13 \mathrm{mEq}$ per $\mathrm{L}$. An average of 85 per cent of the total nitrate infused was recovered in the urine. Organic acid excretion rose slightly in all animals; the cumulative increase above control levels averaged $30 \mathrm{mEq}$.

3. Potassium, sodium, and internal balance (see Table III). Plasma potassium fell from an average of $4.4 \mathrm{mEq}$ per $\mathrm{L}$ at the end of the control period to a final value of $3.0 \mathrm{mEq}$ per $\mathrm{L}$, ranging from 1.9 to 4.1. Potassium losses were generally largest during the first day and tended to diminish thereafter (see Figure 5). The final cumulative potassium losses, corrected for nitrogen, ranged from 64 to $121 \mathrm{mEq}$. The animals remained in approximate sodium balance (see Table III and Figure 5). Calculations of internal balance showed a moderate decrease in the extracellular volume in each animal (average of $0.6 \mathrm{~L}$ ). Calculated intracellular potassium fell by an av- erage of $94 \mathrm{mEq}$ while calculated intracellular sodium rose by an average of $103 \mathrm{mEq}$.

4. Miscellaneous. There was an average weight loss of $0.25 \mathrm{~kg}$. Hematocrit showed no consistent change. Cumulative nitrogen balance was significantly positive in all animals, ranging from +2.0 to $+16.8 \mathrm{~g}$ (see Table III). Plasma creatinine concentration was measured in 7 dogs at the end of the control period and averaged $0.8 \mathrm{mg}$ per cent, ranging from 0.7 to 0.9 . It was again measured in 7 animals at the end of period I, or II, or both; the average value was $0.8 \mathrm{mg}$ per cent, ranging from 0.7 to 0.9 . Plasma creatinine concentration was measured at the end of period III in 2 animals corrected with sodium chloride and in 2 corrected with potassium chloride; the average value was $0.8 \mathrm{mg}$ per cent, ranging from 0.6 to 0.9 . Urine creatinine and urine phosphate remained at control levels throughout the study. Stool losses of electrolytes remained essentially in the control range throughout the study and averaged $4 \mathrm{mEq}$ sodium, $2 \mathrm{mEq}$ potassium, and less than $1 \mathrm{mEq}$ chloride daily. Sulfate excretion was 
TABLE I

Balance data on a dog with chloride deficit repaired by administration

\begin{tabular}{|c|c|c|c|c|c|c|c|c|c|c|c|c|c|c|c|}
\hline & \multirow[b]{2}{*}{ Day } & \multirow[b]{2}{*}{$\begin{array}{c}\text { Body } \\
\text { weight }\end{array}$} & \multicolumn{4}{|c|}{ Intake } & \multicolumn{9}{|c|}{ Urine } \\
\hline & & & $\mathrm{Na}$ & $\mathrm{Cl}$ & $\mathbf{K}$ & $\mathbf{N}$ & Vol & $\mathrm{pH}$ & $\mathrm{HCO}_{3}$ & $\mathrm{Cl}$ & $\mathrm{Na}$ & $\mathbf{K}$ & $\mathrm{NH}_{4}$ & TAt & $\mathrm{PO}_{4}$ \\
\hline \multirow[b]{5}{*}{ Period I } & & kg & $\begin{array}{c}m E q / \\
\text { day }\end{array}$ & $\underset{d a y}{m E q /}$ & $\underset{d a y}{m E q /}$ & $\underset{\text { day }}{g m /}$ & $\begin{array}{l}m l / \\
\text { day }\end{array}$ & & $\underset{\text { day }}{m E q /}$ & $\underset{d a y}{m E q /}$ & $\underset{\text { day }}{m E q /}$ & $\underset{d a y}{m E q /}$ & $\underset{\text { day }}{m E q /}$ & $\underset{d a y}{m E q /}$ & $\begin{array}{c}m M / \\
\text { day }\end{array}$ \\
\hline & 1 & 15.60 & 5 & 5 & 76 & 8.3 & 325 & 6.30 & 1 & 5 & 6 & 63 & 15 & 48 & 44 \\
\hline & 2 & 15.75 & 5 & 5 & 76 & 8.3 & 545 & 5.70 & $\mathbf{0}$ & 4 & 1 & 61 & 36 & 44 & 61 \\
\hline & 3 & 15.60 & 5 & 5 & 76 & 8.3 & 590 & 6.18 & 2 & 5 & 1 & 80 & 27 & 33 & 56 \\
\hline & 4 & 15.60 & 5 & 5 & 76 & 8.3 & 730 & 5.86 & 1 & 2 & 2 & 70 & 31 & 39 & 57 \\
\hline \multirow{4}{*}{$\begin{array}{l}\mathrm{NaNO}_{3} \\
\quad \text { infusions, } \\
165 \mathrm{mM} / \text { day }\end{array}$} & 5 & 15.65 & 175 & 5 & 76 & 8.3 & 1,200 & 6.08 & 8 & 58 & 161 & 103 & 29 & 33 & 52 \\
\hline & 6 & 15.55 & 174 & 4 & 73 & 8.0 & 1,320 & 6.14 & 13 & 40 & 174 & 96 & 33 & 35 & 56 \\
\hline & 7 & 15.30 & 175 & 5 & 75 & 8.2 & 1,400 & 6.24 & 20 & 34 & 197 & 98 & 40 & 34 & 57 \\
\hline & 8 & 15.20 & 170 & 5 & 76 & 8.3 & 1,105 & 6.24 & 12 & 25 & 174 & 87 & 33 & 30 & 50 \\
\hline \multirow{3}{*}{ Period II } & 9 & 15.25 & 170 & 5 & 75 & 8.2 & 1,240 & 6.18 & 10 & 21 & 178 & 96 & 42 & 38 & 60 \\
\hline & 10 & & 4 & 4 & 73 & 8.0 & 840 & 5.96 & 2 & 1 & 5 & 78 & 26 & 37 & 52 \\
\hline & 11 & 15.15 & 5 & 5 & 76 & 8.3 & 730 & 6.10 & 2 & 0 & 5 & 73 & 32 & 36 & 55 \\
\hline & 12 & & 5 & 5 & 76 & 8.4 & 680 & 6.04 & 2 & 1 & 2 & 70 & 33 & 36 & 53 \\
\hline & 13 & 14.75 & 4 & 4 & 72 & 7.9 & 800 & 6.22 & 5 & 1 & 2 & 70 & 34 & 30 & 48 \\
\hline & 14 & 14.85 & 5 & 5 & 75 & 8.2 & 615 & 6.20 & 4 & 1 & 1 & 66 & 31 & 32 & 49 \\
\hline & 15 & 14.65 & 5 & 5 & 74 & 8.2 & 675 & 6.22 & 6 & 1 & 2 & 67 & 30 & 31 & 49 \\
\hline \multirow{6}{*}{$\begin{array}{l}\mathrm{NaCl} \text { supple- } \\
\text { ment }\end{array}$} & 16 & 1490 & 166 & 85 & 0 & 8.2 & 430 & 6.22 & 4 & 1 & 33 & 47 & 26 & 32 & 51 \\
\hline & 17 & 15.00 & 166 & 85 & $\mathbf{0}$ & 8.2 & 460 & 6.67 & 13 & 1 & 71 & 12 & 20 & 16 & 41 \\
\hline & 18 & 15.00 & 162 & 86 & 0 & 8.2 & 650 & 7.39 & 47 & 12 & 141 & 1 & 34 & 0 & 42 \\
\hline & 19 & 14.90 & 162 & 86 & 0 & 8.2 & 700 & 6.68 & 13 & 68 & 169 & 1 & 28 & 20 & 54 \\
\hline & 20 & 14.85 & 161 & 85 & 0 & 8.2 & 520 & 6.53 & 6 & 73 & 142 & 1 & 25 & 19 & 42 \\
\hline & 21 & 14.95 & 164 & 87 & 0 & 8.3 & 605 & 6.49 & 5 & 81 & 147 & 1 & 32 & 21 & 47 \\
\hline \multirow{5}{*}{ Period IV } & 22 & 15.05 & 5 & 5 & 78 & 8.2 & 640 & 6.11 & 3 & 14 & 58 & 3 & 31 & 31 & 49 \\
\hline & 23 & 15.10 & 5 & 5 & 76 & 8.1 & 410 & 5.68 & 1 & 1 & 4 & 21 & 39 & 32 & 43 \\
\hline & 24 & 15.25 & 5 & 5 & 78 & 8.2 & 460 & 6.10 & 2 & 1 & 2 & 50 & 18 & 26 & 41 \\
\hline & 25 & 15.20 & 5 & 5 & 78 & 8.2 & 560 & 6.36 & 7 & 1 & 1 & 69 & 33 & 28 & 51 \\
\hline & 26 & 15.30 & 5 & 5 & 78 & 8.3 & 500 & 6.23 & 4 & 1 & 1 & 81 & 28 & 36 & 60 \\
\hline
\end{tabular}

* Blood was drawn for plasma determinations at the end of each balance day.

† TA = titratable acid.

TABLE II

Balance data on a dog with chloride deficit repaired by administration

\begin{tabular}{|c|c|c|c|c|c|c|c|c|c|c|c|c|c|c|c|}
\hline & \multirow[b]{2}{*}{ Day } & \multirow[b]{2}{*}{$\begin{array}{c}\text { Body } \\
\text { weight }\end{array}$} & \multicolumn{4}{|c|}{ Intake } & \multicolumn{9}{|c|}{ Urine } \\
\hline & & & $\mathrm{Na}$ & $\mathrm{Cl}$ & $\mathbf{K}$ & $\mathrm{N}$ & Vol & $\mathrm{pH}$ & $\mathrm{HCO}_{3}$ & $\mathrm{Cl}$ & $\mathrm{Na}$ & $\mathbf{K}$ & $\mathrm{NH}_{4}$ & TAt & $\mathrm{PO}_{4}$ \\
\hline \multirow{10}{*}{ Period I } & & $k g$ & $\underset{d a y}{m E q /}$ & $\underset{d a y}{m E q /}$ & $\underset{d a y}{m E q /}$ & $\underset{d a y}{g m /}$ & $\begin{array}{l}m l / \\
d a y\end{array}$ & & $\underset{d a y}{m E q /}$ & $\underset{d a y}{m E q /}$ & $\underset{d a y}{m E q /}$ & $\underset{d a y}{m E q /}$ & $\underset{d a y}{m E q /}$ & $\underset{d a y}{m E q /}$ & $\underset{d a y}{m M /}$ \\
\hline & 1 & 12.70 & 3 & 4 & 67 & 7.6 & 285 & 6.25 & 1 & 3 & 1 & 45 & 15 & 17 & 30 \\
\hline & 2 & 12.35 & 3 & 4 & 67 & 7.6 & 740 & 6.36 & 4 & 4 & 1 & 92 & 29 & 34 & 65 \\
\hline & $3 \ddagger$ & 12.95 & 3 & 4 & 67 & 7.6 & 545 & 6.24 & 3 & 1 & 1 & 56 & 21 & 25 & 42 \\
\hline & $4 \ddagger$ & 12.55 & 3 & 4 & 67 & 7.6 & 545 & 6.24 & 3 & 1 & 1 & 56 & 21 & 25 & 42 \\
\hline & 5 & 12.60 & 139 & 4 & 67 & 7.6 & 875 & 6.39 & 12 & 31 & 108 & 76 & 26 & 19 & 36 \\
\hline & 6 & 12.60 & 139 & 4 & 67 & 7.6 & 1,030 & 6.28 & 10 & 25 & 137 & 74 & 31 & 25 & 43 \\
\hline & 7 & 12.60 & 139 & 4 & 67 & 7.6 & 1,030 & 6.40 & 15 & 20 & 142 & 72 & 34 & 22 & 44 \\
\hline & 8 & 12.55 & 139 & 4 & 66 & 7.5 & 860 & 6.35 & 15 & 16 & 134 & 64 & 33 & 20 & 38 \\
\hline & 9 & 12.55 & 139 & 4 & 67 & 7.6 & 830 & 6.42 & 15 & 16 & 135 & 57 & 28 & 18 & 35 \\
\hline \multirow{4}{*}{ Period II } & 10 & 12.40 & 139 & 4 & 67 & 7.6 & 1,105 & 6.33 & 17 & 15 & 133 & 80 & 50 & 31 & 56 \\
\hline & 11 & 12.55 & 137 & 4 & 66 & 7.5 & 855 & 6.37 & 14 & 13 & 134 & 62 & 32 & 20 & 36 \\
\hline & 12 & 12.40 & 3 & 4 & 67 & 7.6 & 630 & 6.30 & 6 & 2 & 15 & 64 & 32 & 33 & 56 \\
\hline & 13 & 12.25 & 3 & 4 & 65 & 7.6 & 445 & 6.31 & 4 & 1 & 5 & 56 & 22 & 25 & 42 \\
\hline \multirow{8}{*}{ Period III } & 14 & 12.35 & 3 & 4 & 65 & 7.6 & 550 & 6.34 & 5 & 1 & 4 & 62 & 21 & 26 & 46 \\
\hline & 15 & 12.35 & 3 & 4 & 65 & 7.6 & 565 & 6.32 & 8 & 1 & 3 & 62 & 31 & 27 & 48 \\
\hline & 16 & 12.25 & 3 & 4 & 65 & 7.6 & 640 & 6.29 & 6 & 1 & 3 & 75 & 33 & 33 & 57 \\
\hline & 17 & 12.45 & 3 & 4 & 65 & 6.9 & 430 & 6.12 & 2 & 1 & 2 & 46 & 21 & 24 & 38 \\
\hline & 18 & 12.50 & 3 & 47 & 108 & 6.8 & 450 & 6.47 & 6 & 1 & 2 & 57 & 19 & 18 & 36 \\
\hline & 19 & 12.30 & 3 & 48 & 109 & 6.9 & 720 & 6.70 & 11 & 2 & 1 & 97 & 17 & 18 & 50 \\
\hline & 20 & 12.30 & 3 & 48 & 109 & 6.9 & 560 & 6.72 & 10 & 26 & 2 & 110 & 17 & 15 & 45 \\
\hline & 21 & 12.35 & 3 & 48 & 109 & 6.9 & 620 & 6.16 & 2 & 43 & 0 & 98 & 23 & 27 & 46 \\
\hline
\end{tabular}

* As in Table I.

$+\mathrm{TA}=$ titratable acid.
$\ddagger$ For technical reasons the urine obtained on these 2 days was pooled. Each recorded value is one-half of the total for the 2 days. 
of sodium chloride and a potassium-free intake $(\operatorname{dog} 0)$

\begin{tabular}{|c|c|c|c|c|c|c|c|c|c|c|c|c|c|c|c|}
\hline \multicolumn{4}{|c|}{ Urine } & \multicolumn{4}{|c|}{ Stool } & \multicolumn{8}{|c|}{ Plasma* } \\
\hline $\begin{array}{l}\text { Organic } \\
\text { acids }\end{array}$ & $\mathrm{NO}_{3}$ & $\begin{array}{c}\text { Creati- } \\
\text { nine }\end{array}$ & $\mathbf{N}$ & $\mathrm{Na}$ & $\mathrm{Cl}$ & $\mathbf{K}$ & $\mathbf{N}$ & $\mathrm{pCO}_{2}$ & $\mathrm{pH}$ & $\mathrm{HCO}_{3}$ & $\mathrm{Na}$ & $\mathrm{Cl}$ & $\mathbf{K}$ & $\mathrm{NO}_{8}$ & Creat. \\
\hline$\underset{d a y}{m E q /}$ & $\underset{d a y}{m E q /}$ & $\underset{d a y}{m g}$ & $\underset{d a y}{g m /}$ & $\underset{d a y}{m E q /}$ & $\underset{\text { day }}{m E q /}$ & $\underset{d a y}{m E q /}$ & $\underset{\text { day }}{g m /}$ & $\underset{H \boldsymbol{g}}{m m}$ & & $\underset{L}{m E q /}$ & $\underset{L}{m E q /}$ & $\underset{L}{m E q /}$ & $\underset{L}{m E q}$ & $\underset{L}{m E q /}$ & $m g \%$ \\
\hline 18 & 2 & 325 & 6.5 & 1 & 1 & 3 & 0.3 & & & & & & & & \\
\hline 22 & 3 & 425 & 10.2 & 1 & 1 & 3 & 0.3 & 36 & 7.37 & 20.4 & 143 & 108 & 4.4 & 0.0 & \\
\hline 25 & 4 & 485 & 10.6 & 1 & 1 & 3 & 0.3 & & & & & & & & \\
\hline 23 & 4 & 415 & 10.1 & 1 & 1 & 3 & 0.3 & 37 & 7.37 & 21.0 & 140 & 106 & 4.8 & & 0.7 \\
\hline 26 & 134 & 390 & 6.5 & 3 & 1 & 0 & 0.4 & 42 & 7.40 & 25.0 & 143 & 99 & 3.8 & 5.0 & 0.8 \\
\hline 28 & 142 & 410 & 5.8 & 3 & 1 & 0 & 0.4 & & & & & & & & \\
\hline 34 & 151 & 495 & 7.4 & 3 & 1 & 0 & 0.4 & 41 & 7.46 & 28.5 & 142 & 87 & 2.6 & 8.0 & 0.7 \\
\hline 23 & 147 & 420 & 6.8 & 3 & 1 & 0 & 0.4 & 39 & 7.48 & 28.4 & 138 & 82 & 2.2 & 9.0 & \\
\hline 28 & 159 & 440 & 8.8 & 3 & 1 & 0 & 0.4 & 42 & 7.50 & 31.8 & 135 & 76 & 1.9 & 8.5 & 0.9 \\
\hline 27 & 11 & 390 & 10.9 & 8 & 1 & 5 & 1.3 & & & & & & & & \\
\hline 25 & 7 & 335 & 9.4 & 8 & 1 & 5 & 1.3 & 51 & 7.44 & 33.6 & 134 & 76 & 2.2 & 5.0 & 0.7 \\
\hline 26 & 5 & 355 & 9.5 & 8 & 1 & 5 & 1.3 & & & $\therefore$ & & & & & \\
\hline 26 & 3 & 350 & 8.4 & 8 & 1 & 5 & 1.3 & 38 & 7.55 & 31.9 & 135 & 75 & 2.9 & & \\
\hline 21 & 3 & 310 & 8.4 & 8 & 1 & 5 & 1.3 & 40 & 7.55 & 33.8 & 136 & 71 & 2.9 & 4.0 & \\
\hline 23 & 1 & 330 & 9.0 & 8 & 1 & 5 & 1.3 & 38 & 7.55 & 32.3 & 133 & 73 & 3.0 & 4.0 & \\
\hline 28 & 4 & 340 & 8.0 & 8 & 1 & 5 & 1.3 & 43 & 7.49 & 32.0 & 136 & 84 & 2.6 & 3.0 & \\
\hline 26 & 5 & 275 & 5.8 & 5 & 1 & 1 & 0.4 & 42 & 7.42 & 26.3 & 143 & 99 & 2.4 & 2.0 & \\
\hline 31 & 8 & 295 & 5.9 & 5 & 1 & 1 & 0.4 & 30 & 7.40 & 18.1 & 144 & 110 & 2.3 & 0.0 & \\
\hline 27 & 5 & 365 & 5.9 & 5 & 1 & 1 & 0.4 & 31 & 7.38 & 17.5 & 146 & 117 & 3.0 & 0.0 & \\
\hline 22 & 3 & 350 & 5.4 & 5 & 1 & 1 & 0.4 & & & & & & & & \\
\hline 21 & 3 & 360 & 5.7 & 5 & 1 & 1 & 0.4 & 33 & 7.35 & 17.4 & 148 & 115 & 2.4 & 0.0 & \\
\hline 17 & 3 & 340 & 5.4 & 5 & 1 & 1 & 0.4 & 31 & 7.40 & 18.9 & 147 & 112 & 4.2 & 0.0 & \\
\hline 17 & 3 & 295 & 5.7 & 5 & 1 & 1 & 0.4 & & & & & & & & \\
\hline 17 & 3 & 250 & 5.0 & 5 & 1 & 1 & 0.4 & & & & & & & & \\
\hline 18 & 4 & 290 & 5.3 & 5 & 1 & 1 & 0.4 & 33 & 7.43 & 21.3 & 148 & 111 & 4.6 & 0.0 & \\
\hline 20 & 5 & 380 & 6.7 & 5 & 1 & 1 & 0.4 & 35 & 7.41 & 21.7 & 148 & 107 & 5.5 & & \\
\hline
\end{tabular}

of potassium chloride and a sodium-free intake $(\operatorname{dog} L)$

\begin{tabular}{|c|c|c|c|c|c|c|c|c|c|c|c|c|c|c|c|}
\hline \multicolumn{4}{|c|}{ Urine } & \multicolumn{4}{|c|}{ Stool } & \multicolumn{8}{|c|}{ Plasma* } \\
\hline $\begin{array}{l}\text { Organic } \\
\text { acids }\end{array}$ & $\mathrm{NO}_{3}$ & $\begin{array}{l}\text { Creati- } \\
\text { nine }\end{array}$ & $\mathbf{N}$ & $\mathrm{Na}$ & $\mathrm{Cl}$ & $\mathbf{K}$ & $\mathbf{N}$ & $\mathrm{pCO}_{2}$ & $\mathrm{pH}$ & $\mathrm{HCO}_{2}$ & $\mathrm{Na}$ & $\dot{\mathrm{Cl}}$ & $\mathbf{K}$ & $\mathrm{NO}_{2}$ & Creat. \\
\hline$\underset{d a y}{m E q /}$ & $\underset{d a y}{m E q /}$ & $\begin{array}{c}m g / \\
d a y\end{array}$ & $\underset{d a y}{g m /}$ & $\underset{d a y}{m E q /}$ & $\underset{d a y}{m E q /}$ & $\underset{d a y}{m E q /}$ & $\underset{\text { day }}{g m /}$ & $\underset{H g}{m m}$ & & $\underset{L}{m E a /}$ & $\underset{L}{m E q /}$ & $\underset{L}{m E q /}$ & $\underset{L}{m E q /}$ & $\underset{L}{m E q /}$ & $m g \%$ \\
\hline 15 & 2 & 330 & 4.7 & 3 & 1 & 4 & 0.7 & & & . & & & & & \\
\hline 30 & 3 & 530 & 9.0 & 3 & 1 & 4 & 0.7 & 37 & 7.38 & 21.2 & 148 & 113 & 4.4 & 0.0 & 0.9 \\
\hline 17 & 2 & 305 & 5.4 & 3 & 1 & 4 & 0.7 & & & & & & & & \\
\hline 17 & 2 & 305 & 5.4 & 3 & 1 & 4 & 0.7 & 34 & 7.40 & 20.6 & 146 & 112 & 4.6 & 0.0 & 0.8 \\
\hline 21 & 91 & 360 & 4.6 & 3 & 1 & 0 & 0.4 & 35 & 7.43 & 22.5 & 146 & 103 & 4.0 & 5.0 & 0.8 \\
\hline 22 & 123 & 360 & 4.4 & 3 & 1 & 0 & 0.4 & & & & & & & & \\
\hline 24 & 129 & 370 & 4.6 & 3 & 1 & 0 & 0.4 & 42 & 7.38 & 24.3 & 145 & 96 & 3.1 & 6.0 & 0.8 \\
\hline 23 & 125 & 370 & 4.6 & 3 & 1 & $\mathbf{0}$ & 0.4 & & & & & & & & \\
\hline 24 & 118 & 320 & 4.4 & 3 & 1 & 0 & 0.4 & 42 & 7.41 & 25.9 & 143 & 92 & 2.7 & 8.0 & \\
\hline 28 & 126 & 420 & 6.4 & 3 & 1 & 0 & 0.4 & & & & & & & & \\
\hline 22 & 125 & 340 & 4.4 & 3 & 1 & 0 & 0.4 & 38 & 7.46 & 26.4 & 143 & 86 & 2.8 & 9.0 & \\
\hline 30 & 10 & 440 & 8.0 & 2 & 1 & 1 & 0.8 & 40 & 7.48 & 28.8 & 139 & 86 & 2.6 & 7.0 & \\
\hline 18 & 3 & 305 & 6.2 & 2 & 1 & 1 & 0.8 & & & & & & & & 、 \\
\hline 20 & 1 & 345 & 6.7 & 2 & 1 & 1 & 0.8 & 43 & 7.45 & 28.8 & 141 & 89 & 3.0 & 4.5 & \\
\hline 18 & 1 & 350 & 6.5 & 2 & 1 & 1 & 0.8 & & & & & & & & \\
\hline 23 & 2 & 395 & 7.7 & 2 & 1 & 1 & 0.8 & 49 & 7.41 & 30.2 & 141 & 86 & 3.3 & 2.5 & \\
\hline 16 & 2 & 290 & 5.5 & 2 & 1 & 1 & 0.8 & 45 & 7.46 & 31.3 & 143 & 86 & 3.7 & & 0.8 \\
\hline 16 & 2 & 280 & 5.6 & 3 & 1 & 2 & 0.6 & 39 & 7.45 & 26.3 & 143 & 96 & 5.1 & 2.0 & \\
\hline 32 & 4 & 405 & 8.5 & 3 & 1 & 2 & 0.6 & 32 & 7.37 & 17.9 & 143 & 105 & 5.2 & 0.5 & 0.9 \\
\hline 28 & 6 & 345 & 6.4 & 3 & 1 & 2 & 0.6 & 31 & 7.37 & 17.5 & 142 & 108 & 4.7 & 0.0 & \\
\hline 17 & 3 & 330 & 6.3 & 3 & 1 & 2 & 0.6 & 35 & 7.34 & 18.1 & 139 & 110 & 5.2 & & 0.8 \\
\hline
\end{tabular}




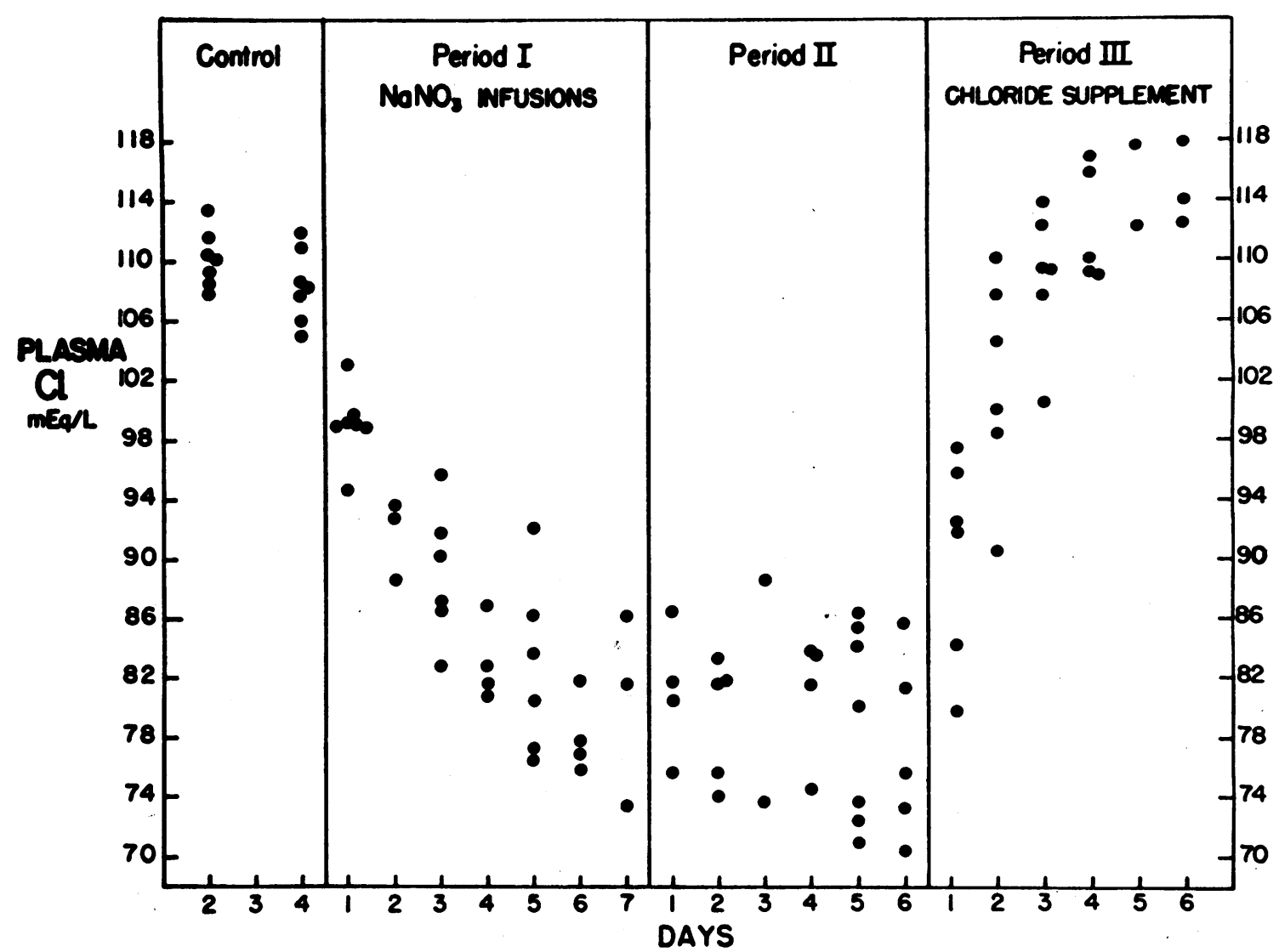

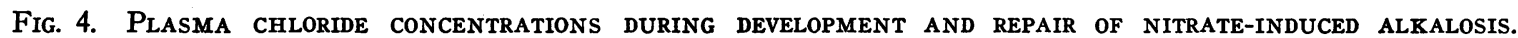
For details of protocols employed for the provision of the chloride supplements in period III, see text. The period of $\mathrm{KHCO}_{3}$ administration in dog $\mathrm{I}$ is omitted.

measured in 2 animals and showed no significant change throughout the study.

\section{Period II}

1. Acid-base balance. Plasma bicarbonate concentration continued to rise slowly. The average increment was $2.0 \mathrm{mEq}$ per $\mathrm{L}$, with a total average rise from control of $11 \mathrm{mEq}$ per L. Plasma $\mathrm{pH}$ and $\mathrm{pCO}_{2}$ remained elevated. Net acid exretion showed no consistent deviation from control levels.

2. Chloride, nitrate, unmeasured anions, and organic acids. As shown in Figures 1, 2, and 4, plasma chloride showed no significant changes. Urine chloride output was negligible. Plasma nitrate fell steadily in all animals (see Figures 1, 2, and 6), reaching final values, before chloride administration, of 3 to $8 \mathrm{mEq}$ per $\mathrm{L}$. By the second day urine nitrate excretion had fallen to control levels. The reduction in plasma nitrate concentration was accompanied by an equivalent rise in bicarbonate, or unmeasured anion concentration, or both (see Figure 6). Organic acid excretion was only slightly above control levels. Dog I, which received a supplement of potassium bicarbonate over 4 days, showed no significant potassium retention or change in plasma acid-base status.

3. Potassium, sodium, and internal balance (see Table III). There was no significant change in plasma sodium and potassium concentration. Average cumulative potassium balance, corrected for nitrogen, was $+14 \mathrm{mEq}$ and average cumulative sodium balance was $-32 \mathrm{mEq}$.

4. Miscellaneous. Body weight fell by an average of $0.75 \mathrm{~kg}$. Hematocrit remained stable. Nitrogen balance was slightly negative in all animals.

Period III. Administration of chloride supplement

1. Acid-base balance. The general pattern of changes was similar in all animals regardless of the cation with which chloride was given (see 

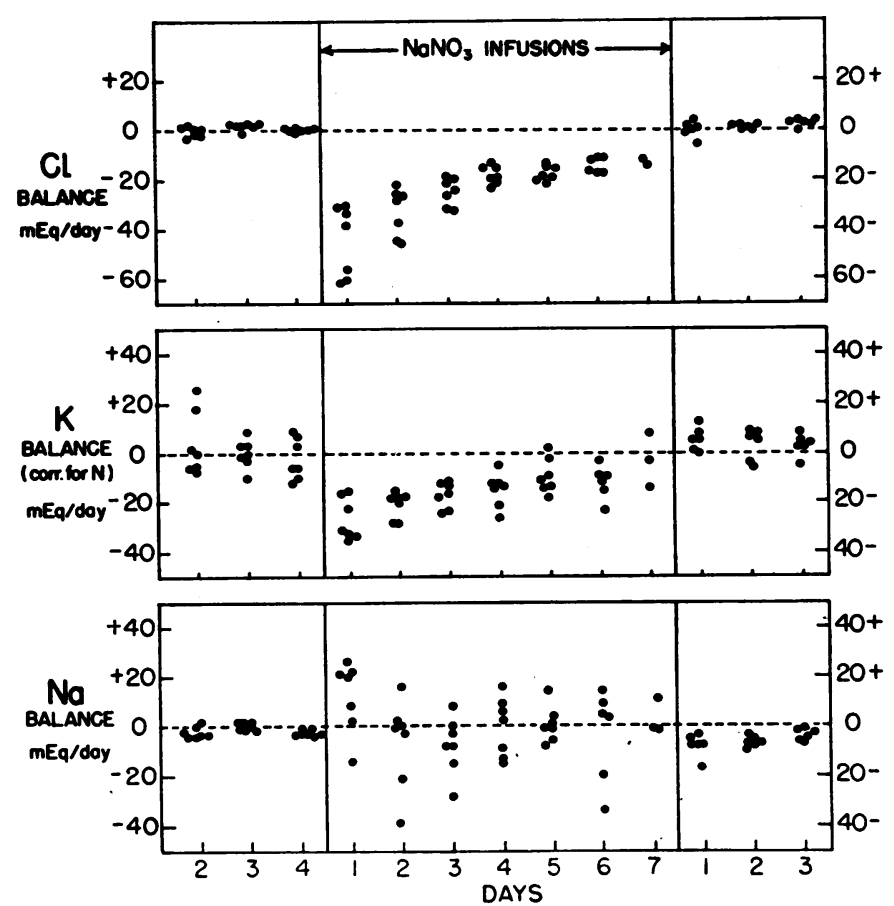

Fig. 5. DaIly balances (NON-Cumulative) fOR Chloride, POTASSIUM, AND SODIUM DURING AND AFTER SODIUM NITRATE INFUsions. The values for potassium balance have been corrected for nitrogen balance $(2.7 \mathrm{mEq}$ per $\mathrm{g}$ ).

TABLE III

Changes in electrolyte balance *

\begin{tabular}{|c|c|c|c|c|c|c|c|c|c|c|c|c|c|}
\hline & \multirow[b]{4}{*}{ Dog } & \multirow[b]{4}{*}{ Days } & \multirow{3}{*}{\multicolumn{2}{|c|}{ Weight }} & \multirow{2}{*}{\multicolumn{6}{|c|}{ External balance }} & \multicolumn{3}{|c|}{ Internal balance } \\
\hline & & & & & & & & & & & \multirow{3}{*}{$\begin{array}{l}\text { Intra- } \\
\text { cellular } \\
\mathrm{Na}\end{array}$} & \multirow{3}{*}{$\begin{array}{c}\text { Intra- } \\
\text { cellular } \\
\mathrm{K \dagger}\end{array}$} & \multirow{3}{*}{$\begin{array}{c}\text { Extra- } \\
\text { cell. } \\
\text { fluid } \\
\text { vol }\end{array}$} \\
\hline & & & & & & & $\mathrm{Cl}-\frac{\mathrm{Na}}{2 \mathrm{a}}$ & & $\mathbf{K}$ corr. & & & & \\
\hline & & & Initial & Final & $\mathrm{Cl}$ & $\mathrm{Na}$ & & $\mathbf{K}$ & for $\mathrm{N} \dagger$ & $\mathbf{N}$ & & & \\
\hline \multicolumn{14}{|l|}{ Period I } \\
\hline \multirow{7}{*}{$\begin{array}{l}\mathrm{NaNO}_{2} \\
\text { infusions }\end{array}$} & $\mathrm{K} \boldsymbol{t}$ & 6 & 17.25 & 16.65 & -194 & -21 & -178 & -115 & -121 & +2.0 & +123 & -112 & -0.8 \\
\hline & $\mathbf{P}$ & 7 & 18.00 & 17.75 & -133 & -3 & -131 & -99 & -115 & +5.7 & +69 & -113 & -0.3 \\
\hline & $\mathbf{F}$ & 7 & 13.60 & 13.55 & -152 & -12 & -143 & -78 & -100 & +8.0 & +102 & -93 & -0.7 \\
\hline & G & 6 & 16.65 & 16.30 & -180 & -47 & -144 & -81 & -106 & +9.3 & +96 & -102 & -0.8 \\
\hline & O & 5 & 15.60 & 15.25 & -167 & 0 & -167 & -105 & -115 & +3.7 & +123 & -105 & -0.8 \\
\hline & I & 6 & 11.60 & 11.60 & -115 & +33 & -140 & -45 & -81 & +13.3 & +103 & -77 & -0.4 \\
\hline & L & 7 & 12.55 & 12.55 & -123 & +19 & -138 & -18 & -64 & +16.8 & +106 & -58 & -0.5 \\
\hline \multirow{5}{*}{ Period II } & $P \ddagger$ & 6 & 17.50 & 17.30 & +16 & -29 & +38 & -11 & +10 & -7.6 & -58 & +8 & +0.2 \\
\hline & $\mathbf{F}$ & 6 & 13.55 & 13.45 & +3 & -33 & +28 & +4 & +18 & -5.2 & -38 & +17 & +0.1 \\
\hline & G & 5 & 16.30 & 16.15 & +8 & -24 & +27 & -12 & -6 & -2.1 & -22 & -3 & 0.0 \\
\hline & 0 & 6 & 15.25 & 14.65 & +9 & -46 & +44 & -9 & +31 & -14.4 & -71 & +28 & +0.2 \\
\hline & I\& & 5 & 11.60 & 11.25 & o & -20 & +15 & -5 & +8 & -4.9 & +3 & +10 & -0.1 \\
\hline \multicolumn{14}{|l|}{ Period III } \\
\hline \multirow{6}{*}{$\begin{array}{l}\text { Chloride } \\
\text { supplement }\end{array}$} & $\mathbf{F}$ & 6 & 13.45 & 14.30 & +361 & +294 & +135 & -52 & -85 & +11.7 & -47 & -89 & +2.1 \\
\hline & G & 6 & 16.15 & 16.80 & +303 & +246 & +114 & -76 & -108 & +11.4 & -15 & -116 & +1.6 \\
\hline & 0 & 6 & 14.65 & 14.95 & +262 & +228 & +87 & -73 & -98 & +9.3 & +15 & -99 & +1.1 \\
\hline & I & 4 & 11.35 & 11.10 & +96 & -21 & +112 & +102 & +95 & +2.5 & -96 & +89 & +0.5 \\
\hline & $\mathbf{L}$ & 4 & 12.45 & 12.35 & +107 & -17 & +120 & +67 & +71 & -1.7 & -71 & +54 & +0.4 \\
\hline & $\mathbf{E}$ & 4 & 12.90 & 13.40 & +224 & +127 & +126 & +69 & +51 & +6.6 & -51 & +60 & +0.8 \\
\hline \multirow{2}{*}{ Period IV } & G & 6 & 16.80 & 16.85 & -42 & -108 & +41 & +165 & +138 & +10.0 & -113 & +131 & +0.1 \\
\hline & O & 5 & 14.95 & 15.30 & -4 & -76 & +54 & +159 & +129 & +10.9 & -112 & +116 & +0.2 \\
\hline
\end{tabular}

* The cumulative balances refer only to the changes that occurred during the individual period.

† Correction for $\mathrm{N}=2.7 \mathrm{mEq}$ potassium per gram of nitrogen.

Study was discontinued because of diarrhea that began after completion of this period.

The KHCOs supplement period is not included. 
Figures 1-3). There was a large decline in plasma bicarbonate concentration to a final average level of $18.0 \mathrm{mEq}$ per $\mathrm{L}$, with an average total decrement of $14.5 \mathrm{mEq}$ per $\mathrm{L}$. At the same time $\mathrm{pH}$ and $\mathrm{pCO}_{2}$ fell to normal. Cumulative delta net acid excretion ranged from -71 to $-174 \mathrm{mEq}$, averaging -127 . In the animals receiving sodium chloride and a potassium-free intake, a significant portion of the decrement in net acid excretion was the result of a bicarbonate diuresis (see Table I) which accounted cumulatively for 58,44 , and $43 \mathrm{mEq}$, respectively. There was a delay of 1 day before plasma bicarbonate began to fall in the animals receiving sodium chloride.

2. Chloride, nitrate, unmeasured anions, and organic acids. As shown in Figures 1, 2, and 4, there was a rapid restoration to normal of plasma chloride, with an average final chloride concentration of $113 \mathrm{mEq}$ per $\mathrm{L}$. The total "selective" chloride gains, corrected for sodium retention in animals given sodium chloride, ranged from 87 to $135 \mathrm{mEq}$ (see Table III). Plasma nitrate fell to undetectable levels and unmeasured anions fell to or below control values (see Figure 6). Organic acid excretion remained essentially at control levels.

3. Potassium, sodium, and internal balance (see Table III). Protocol a) $\mathrm{NaCl}$. During the first 2 days there was an average total loss of $65 \mathrm{mEq}$ potassium, corrected for nitrogen, unaccompanied by any significant decrease in plasma potassium concentration. By the third day after potassium withdrawal, urine potassium output was $1 \mathrm{mEq}$ or less per day. Cumulative sodium retention averaged $309 \mathrm{mEq}$, and plasma sodium rose by an average of $9 \mathrm{mEq}$ with an increase in calculated extracellular volume of $1.6 \mathrm{~L}$. Calculated intracellular sodium did not change significantly. Protocol b) $\mathrm{KCl}$. An average of $83 \mathrm{mEq}$ of potassium, corrected for nitrogen, was retained and
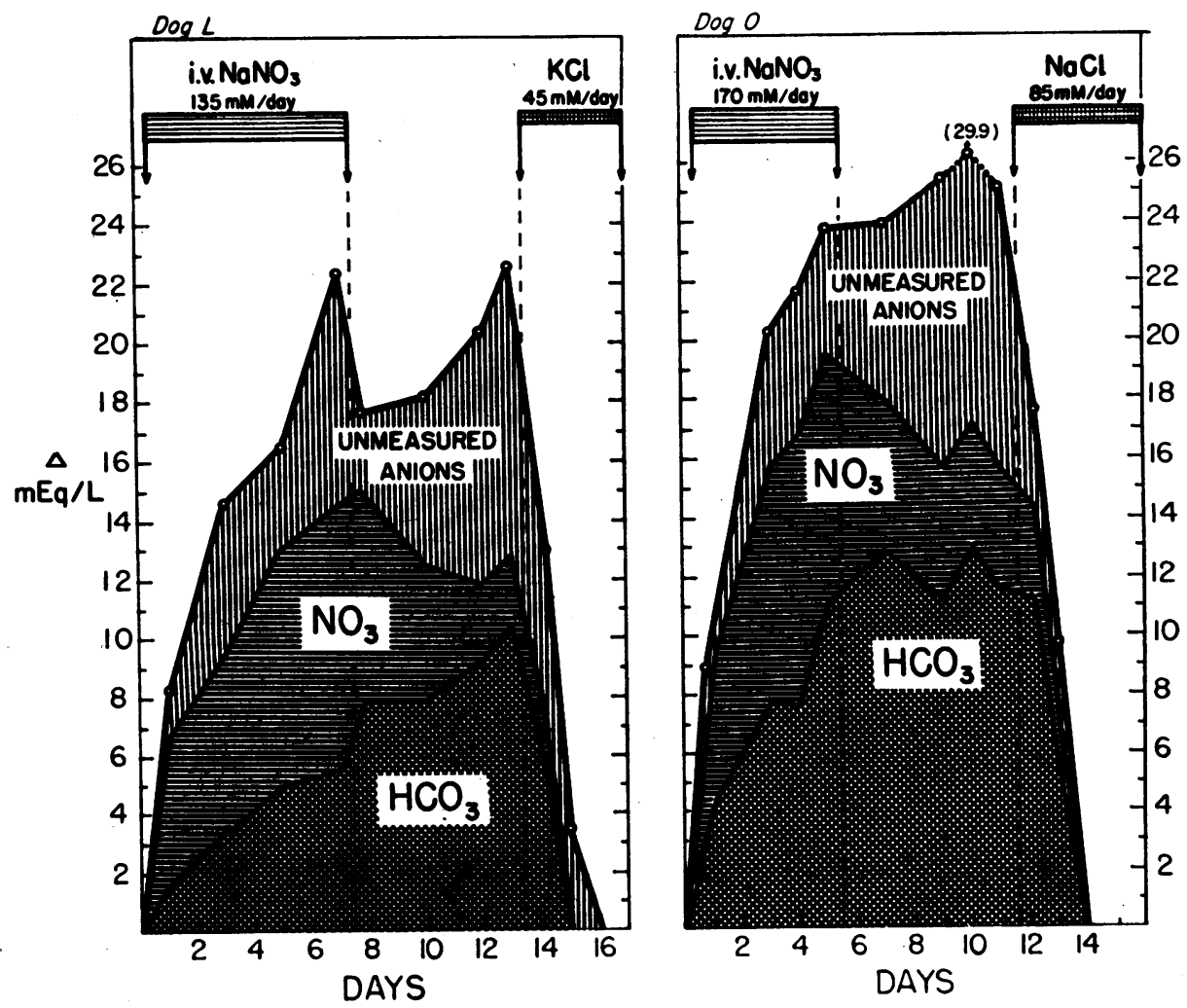

Fig. 6. Changes in plasma concentrations of bicarbonate, nitrate, and unmeasured ANIONS IN TWO REPRESEntative studies. The changes are plotted additively so that the upper line represents $\Delta \mathrm{HCO}_{8}+\Delta \mathrm{NO}_{3}+\Delta$ unmeasured anions. This sum is approximately equal to the fall in plasma chloride concentration since plasma $\left[\mathrm{Na}^{+}\right]$showed only slight changes. 
plasma potassium concentration rose to normal levels. There was no significant change in sodium balance and plasma sodium concentration was unchanged. Calculated loss of intracellular sodium averaged $84 \mathrm{mEq}$ and extracellular volume expanded by an average of $0.5 \mathrm{~L}$. Protocol c) $\mathrm{NaCl}$ and $\mathrm{KCl}$. Moderate retention of both sodium and potassium occurred and all plasma electrolyte concentrations returned to normal. Internal balance calculations are shown in Table III.

4. Miscellaneous. Animals receiving sodium chloride, on protocols a) and c), gained an average of $0.75 \mathrm{~kg}$ with an average fall in hematocrit from 47 to 39 per cent. The animals given only potassium chloride, on protocol $\mathrm{b}$ ), showed a slight loss of weight and no change in hematocrit. Nitrogen balance was positive in the 4 animals receiving sodium (see Table III).

\section{Period IV}

Two of the animals given sodium chloride and a potassium-free diet during period III were returned to the low-chloride, high-potassium diet used in periods I and II. The animals retained an average of $134 \mathrm{mEq}$ of potassium, and plasma potassium concentration returned to control levels. Plasma bicarbonate concentration rose slightly. Net acid excretion returned to the levels observed prior to the administration of chloride. Nitrogen balance was significantly positive (see Table III).

\section{DISCUSSION}

It is noteworthy that production of severe chloride depletion by means of sodium nitrate infusions was not accompanied by a net loss of sodium despite an average decrease in plasma chloride concentration of $29 \mathrm{mEq}$ per L. Only part of the reduction in chloride concentration was compensated for by a rise in plasma nitrate, and even this replacement was transient, plasma nitrate concentration falling to a low level within a few days after the infusions were discontinued. ${ }^{2}$ The most remarkable change in plasma composition was a rise in bicarbonate concentration which averaged

\footnotetext{
2 It is of interest that part of the hypochloremia was compensated for by a rise in unmeasured anions. Since these anions have not been identified, it does not seem fruitful to speculate on the mechanisms responsible for the rise.
}

$11 \mathrm{mEq}$ per L. Body fluid composition was further altered by a loss of potassium which led to an average cumulative deficit of $100 \mathrm{mEq}$. Although equilibrium between potassium intake and excretion was achieved once nitrate infusions were discontinued, it is noteworthy that there was no significant correction of the previous deficit de- spite a continued high potassium intake.

The present data are compatible with the hypothesis that the progressive loss of chloride was responsible for the changes in body fluid composition induced by the nitrate infusions. The possibility must be considered, however, that factors other than the anion deficit were responsible for these alterations. For example, a reduction in glomerular filtration rate, secondary to changes in extracellular volume or to a direct effect of nitrate on the kidney, might be invoked to explain the elevated bicarbonate threshold. There is, however, no evidence of any change in filtration rate; plasma creatinine concentration did not change significantly either during or after the period of nitrate infusions. It is recognized, of course, that plasma creatinine is a relatively insensitive index of changes in filtration rate; but, even if filtration fell slightly, it still seems doubtful that such a fall could account for the rise in bicarbonate threshold. Although it is well known that a reduction of filtration rate induced acutely by renal artery constriction increases the rate of bicarbonate reabsorption per unit volume of filtrate ( 7$)$, there is no evidence that a chronic reduction leads to a similar change; moreover, wide variations in filtration rate induced by fasting or by feeding meat do not alter bicarbonate reabsorptive capacity per $100 \mathrm{cc}$ of glomerular filtrate $(7,8)$.

It also might be proposed that the potassium deficit was the sole factor responsible for the rise in bicarbonate threshold. This explanation, however, seems unlikely, since the threshold for bicarbonate fell with the administration of sodium chloride despite the fact that potassium had been withdrawn from the diet. In fact, a further loss of potassium, approximately equal in magnitude to that which had occurred during development of the alkalosis, failed to prevent rapid restoration of normal acid-base equilibrium. The possibility remains, however, that the state of renal potassium 
stores varied independently of the over-all potassium balance and was critical to the changes in renal threshold for bicarbonate. If such were the case, it would appear, as discussed later, that the changes in tubular cell potassium were determined by the availability of chloride.

The present data allow no definite conclusion concerning the origin of the alkali that appeared in body fluids. ${ }^{3}$ If it is assumed that the endogenous acid load remained constant as the plasma bicarbonate concentration rose, the absence of a rise in net acid excretion would indicate that the generation of bicarbonate was extrarenal. The fact that potassium balance was negative over this interval favors the possibility that tissue accumulation of hydrogen was responsible for the alkali increment. Several observations during and subsequent to correction of the alkalosis, however, are difficult to reconcile with such an hypothesis. Repair of the potassium deficit was apparently not accompanied by release of hydrogen from the intracellular fluid; during administration of potassium chloride the suppression of net acid excretion was more than adequate to account for the fall in extracellular bicarbonate concentration. During administration of sodium chloride, at a time when the potassium deficit was becoming increasingly severe, net acid excretion was suppressed by an amount that would account for a reduction in alkali concentration of 15 mmoles per $\mathrm{L}$ through a volume larger than total body water. It is conceivable that sequestration of hydrogen in cells as a result of the further loss of potassium that occurred during this time contributed to the decrease in acid excretion. Such an interpretation, however, seems unlikely, since subsequent repair of the potassium deficit led neither to a fall in plasma bicarbonate concentration nor to an augmentation of net acid excretion. ${ }^{4}$

\footnotetext{
3 It should be noted that contraction of the extracellular fluid volume, as estimated from "chloride space" calculations, could account in part for the $11 \mathrm{mEq}$ per $\mathrm{L}$ rise in bicarbonate concentration. Even if it is assumed, however, that the alkalosis was limited to the extracellular compartment, only half of the increment in bicarbonate concentration (average 46 per cent; range 8 to 69 per cent) could be explained in this way.

4 It should be noted, incidentally, that in the dog, in contrast to the rat (9), there is no evidence that potassium depletion leads to significant uptake of hydrogen by tissues; during the repair of severe potassium deficiency in-
}

From the considerations above it is apparent that no conclusion concerning the genesis of the alkali increment can be confidently reached at this time. It should be pointed out, however, that whatever the origin of the additional bicarbonate, the alkalosis that followed the infusion of nitrate could have developed only if the renal threshold for bicarbonate had been increased.

There are several considerations favoring the hypothesis that selective depletion of chloride was primarily responsible for the rise in bicarbonate threshold and the development of a negative potassium balance. ${ }^{5}$ It has already been demonstrated in acute studies $(1,10)$ that a disproportion between sodium reabsorption and availability of reabsorbable anion can increase the fraction of sodium reabsorbed through exchange with potassium and hydrogen. It seems reasonable to suggest that such an increase might well be engendered and sustained by a chronic deficit of reabsorbable anion. The observation that in the present studies administration of chloride was the factor critical to correction of both the alkalosis and the potassium deficit accords with this view. During the provision of sodium chloride and a potassium-free diet there was rapid restoration of normal acid-base equilibrium and a striking suppression of net acid excretion. Although the fall in plasma concentration theoretically could be accounted for by expansion of the extracellular fluid, it is clear from data on bicarbonate excretion that the kidney did, in fact, play a critical role in the corrective process. Bicarbonate excretion increased in each animal, the cumulative increment averaging $48 \mathrm{mEq}$; it can readily be calculated that retention of such an amount of bicarbonate would have more than offset the dilution effect. Since this increase in bicarbonate excretion occurred at

duced by Doca, alkali excretion by the kidney fully accounts for the reductions in bicarbonate concentration (3).

5 It is conceivable that the cumulative negative potassium balance resulted simply from administration of a sodium load to animals that had previously been sodium restricted. However, administration on successive days of equimolar amounts of sodium chloride rather than sodium nitrate does not produce a significant potassium deficit (unpublished data). The development of potassium deficiency appears, therefore, to be determined by the nature of the administered anion rather than by the sodium load. 
the time when plasma level was falling sharply (see Table I), it appears that provision of chloride led to restoration of a normal threshold for bicarbonate. Prompt correction also occurred during administration of potassium chloride and a sodium-free intake, although the alkalosis was resistant to the administration of potassium as phosphate.

Just as administration of chloride was essential to correction of the alkalosis, so it was essential to correction of the potassium deficit. After discontinuation of the nitrate infusions, potassium losses were not repaired despite continued provision of large quantities of potassium in the diet. In contrast, the potassium deficit was readily corrected either during or after chloride repletion.

Although, within the theoretical framework above, chloride deficiency may be viewed as independently affecting renal hydrogen and potassium secretion, the possibility remains that chloride deficiency increased the bicarbonate threshold through its effect on tubular potassium stores. The lowering of the renal threshold for bicarbonate in the face of an increasing potassium deficit would, at first, seem incompatible with such a possibility. It is conceivable, however, that the provision of chloride allowed a reaccumulation of potassium within renal tubular cells despite doubling of the over-all deficit, and that such a reaccumulation mediated the correction of the alkalosis. Similarly, the refractoriness of the alkalosis to the administration of potassium phosphate after the nitrate infusions were discontinued could conceivably have resulted from an inability of the tubular cells to replenish their potassium stores in the face of chloride deficiency. In any event, it appears that the availability of chloride was the final determinant of the observed changes in renal threshold for bicarbonate. Such a role of chloride depletion could also account for the unexplained rises in plasma bicarbonate concentration previously noted after intravenous administration of sodium nitrate (11) or peritoneal dialysis with sodium nitrate (12).

\section{SUMMARY}

Chloride depletion was induced in 8 dogs, on a low-chloride diet, by the infusion of 11 mmoles of sodium nitrate per $\mathrm{kg}$ of body weight on 5 to 7 successive days. During this period there was no net loss of sodium nor any significant fall in serum sodium concentration. There was, however, a progressive fall in chloride concentration that was accompanied by an increase in the plasma levels of nitrate, bicarbonate, and unmeasured anions, and also by a negative potassium balance. When nitrate infusions were discontinued, plasma nitrate concentration fell and plasma bicarbonate rose slightly to final levels averaging $11 \mathrm{mEq}$ per $\mathrm{L}$ above control. During the post-infusion period the continued administration of a large quantity of potassium failed to correct either the alkalosis or the potassium deficit. Although the present data do not define the origin of the additional bicarbonate that appeared in the extracellular fluid, they indicate that there was a marked rise in the renal threshold for bicarbonate.

Correction of the metabolic alkalosis was rapidly effected by the administration of sodium chloride, despite the fact that simultaneous withdrawal of potassium from the diet resulted in a marked increase in the severity of the potassium deficit. Since the fall in plasma bicarbonate concentration was accompanied by a substantial increase in bicarbonate excretion, it appears that the renal threshold for bicarbonate had been returned to normal. This finding, together with the observation that administration of potassium chloride and a sodium-free diet also promptly restored acid-base equilibrium, indicates that provision of chloride was the factor critical to the corrective process. Similarly, it was shown that the potassium deficit could be repaired only when chloride stores were replenished.

On the basis of these observations, it is tentatively proposed that chloride deficiency was responsible for both the increase in bicarbonate threshold and the negative potassium balance. It is suggested that the loss of chloride, by reducing the availability of reabsorbable anion, increased the fraction of sodium reabsorbed by means of cation exchange. Although, within the theoretical framework above, chloride deficiency may be viewed as independently affecting renal hydrogen and potassium secretion, the possibility remains that chloride deficiency increased the bicarbonate threshold through its effect on tubular potassium stores. Such a proposal would imply that sodium chloride administration allowed local reaccumula- 
tion of tubular cell potassium despite the increasing negative potassium balance. In any event, it appears that the availability of chloride was the final determinant of the observed changes in renal threshold for bicarbonate.

\section{REFERENCES}

1. Bank, N., and Schwartz, W. B. The influence of anion penetrating ability on urinary acidification and the excretion of titratable acid. $J$. clin. Invest. 1960, 39, 1516.

2. Schwartz, W. B., Hays, R. M., Polak, A., and Haynie, G. D. Effects of chronic hypercapnia on electrolyte and acid-base equilibrium. II. Recovery, with special reference to the influence of chloride intake. J. clin. Invest. 1961, 40, 1238.

3. Atkins, E. L., and Schwartz, W. B. Factors governing correction of the alkalosis associated with potassium deficiency; the critical role of chloride in the recovery process. J. clin. Invest. 1962, 41, 218.

4. Rapoport, S., and West, C. D. Ionic antagonism: Effect of various anions on chloride excretion during osmotic diuresis in the dog. Amer. J. Physiol. 1950, 162, 668.
5. Engelbrecht, R. M., and McCoy, F. A. Rapid determination of nitrate nitrogen in the presence of ammonia and urea. Analyt. Chem. 1956, 28, 1619.

6. Peters, J. P., and Van Slyke, D. D. Quantitative Clinical Chemistry. Baltimore, Williams and Wilkins, 1932, vol. 2, p. 892.

7. Thompson, D. D., and Barrett, M. J. Renal reabsorption of bicarbonate. Amer. J. Physiol. 1954, 176, 201.

8. Pitts, R. F., and Lotspeich, W. D. Bicarbonate and the renal regulation of acid base balance. Amer. J. Physiol. 1946, 147, 138.

9. Cooke, R. E., Segar, W. E., Cheek, D. B., Coville, F. E., and Darrow, D. C. The extrarenal correction of alkalosis associated with potassium deficiency. J. clin. Invest. 1952, 31, 798.

10. Schwartz, W. B., Jenson, R. L., and Relman, A. S. Acidification of the urine and increased ammonium excretion without change in acid-base equilibrium: Sodium reabsorption as a stimulus to the acidifying process. J. clin. Invest. 1955, 34, 673.

11. Hiatt, E. P. Extreme hypochloremia in dogs induced by nitrate administration. Amer. J. Physiol. 1940, 129, 597.

12. Holliday, M. A. Acute metabolic alkalosis: Its effect on potassium and acid excretion. J. clin. Invest. $1955,34,428$. 